\title{
Comparison of Stochastic Forecasting Models
}

\author{
Shalin Shah \{sshah100@jhu.edu\} \\ Stochastic Differential Equations Course Project \\ Johns Hopkins University
}

\begin{abstract}
In this work, we compare several stochastic forecasting techniques like Stochastic Differential Equations (SDE), ARIMA, the Bayesian filter, Geometric Brownian motion (GBM), and the Kalman filter. We use historical daily stock prices of Microsoft (MSFT), Target (TGT) and Tesla (TSLA) and apply all algorithms to try to predict 54 days ahead. We find that there are instances in which all algorithms do well, or do poorly. We find that all three stocks have a strong auto-correlation and a high Hurst factor which shows that it is possible to predict future prices based on a short history of past prices. In the geometric Brownian motion model, there are two parameters for drift and diffusion which are not time dependent. In our more general SDE model (TDNGBM), we have time-dependent drift and time-dependent diffusion terms which makes it more effective than GBM. We measure all algorithms on the correlation between the predicted and actual values, and the mean absolute error (MAE) and we also report confidence intervals generated by the methods. Confidence intervals are more important than point forecasts, and we see that TDNGBM and ARIMA produce good bounds.
\end{abstract}

Keywords: Stochastic Differential Equations, Stochastic forecasting, time series analysis, Kalman filter, ARIMA, stock price prediction, stock market forecasting, Bayesian filter

\section{Introduction}

Stochastic differential equations (SDE) [1] are used to model the dynamics of systems, like stock prices, population growth models, demand forecasting and for stochastic control systems. They also find applications in physics and engineering. An SDE can be used for forecasting, and as this work shows, it can be effective. An SDE uses Brownian motion (zero mean, stationary increments and Gaussian process) to model the stochastic variation of the quantities of interest. Brownian motion is a stochastic process, a Gaussian process, in which the $t^{t h}$ 
Table 1: Hurst Coefficients

\begin{tabular}{ll}
\hline Stock & Hurst Coefficient \\
\hline TGT & 0.84 \\
MSFT & 0.85 \\
TSLA & 0.85
\end{tabular}

instance $B_{t}$ is modeled as $N(0, t)$. The variance increases as the time increases. For $0 \leq s \leq t, B_{t}-B_{s}$ is modeled as $N(0, t-s)$.

In this work we compare several stochastic forecasting algorithms for forecasting stock prices [2] with a 54 days horizon. We first analyze the data using ACF and PACF plots and also the Hurst factor [3. The Hurst factor (or Hurst exponent) measures the magnitude of auto-correlation present in the series. We see that all three data sets (MSFT, TSLA and TGT) exhibit strong auto-correlation, but also a random component (modeled using Brownian motion).

We compare ARIMA, two SDE based algorithms [4] [1] [5], a Bayesian filter [6] [7, and the Kalman filter [8. We find that after proper tuning, there are cases for which some algorithms do well and some algorithms do poorly (more in the results section).

The code is available in the following git repository:

https://github.com/shah314/forecasting

\section{The Data}

We took 253 days of stock price history (2020-06-24 to 2021-06-24) of Microsoft (MSFT), Tesla (TSLA) and Target (TGT). Out of these 253 days the first 199 days are used for training and the rest of the 54 days are used as a test set on which we present the results. So, for all algorithms, the time forecast horizon is 54 days.

The following are the ACF and PACF plots of all three stocks. There is a strong AR component to these time series with lag 1 having the largest autocorrelation. 


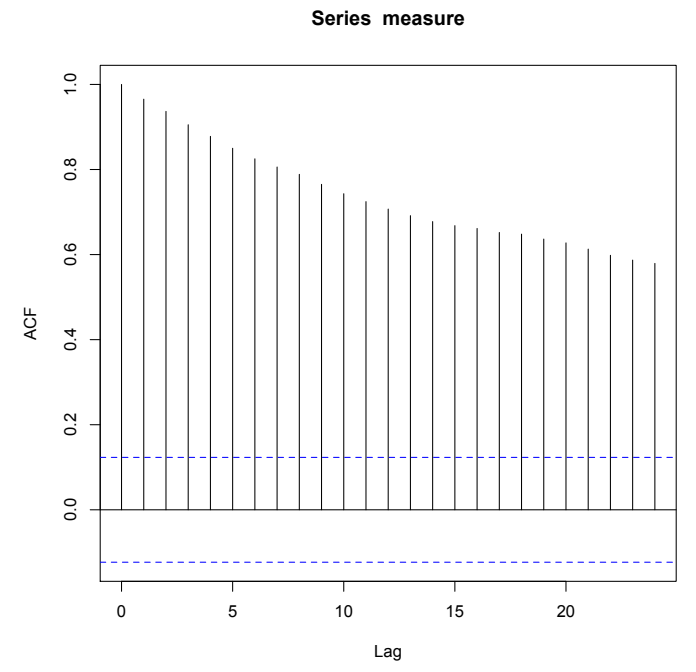

Figure 1: MSFT ACF Plot

\section{Methods}

\subsection{Geometric Brownian Motion (GBM)}

Geometric Brownian Motion (GBM) 44 [1 [5] 2] is a stochastic differential equation (SDE) with time-independent drift and diffusion parameters. The GBM $\mathrm{SDE}$ is given by the following equation:

$d X_{t}=\mu X_{t} d t+\sigma X_{t} d B_{t}$

Where $B_{t}$ is Brownian Motion, $\mu$ is the drift coefficient and $\sigma$ is the volatility coefficient. The parameters $\mu$ and $\sigma$ can be learned using maximum likelihood estimation (MLE) techniques. We use the Sim.DiffProc R package to learn and simulate from the SDE.

This equation can be solved exactly and the solution is as under:

$X_{t}=X_{0} \exp \left(\left(\mu-\frac{\sigma^{2}}{2}\right) t+\sigma W_{t}\right)$

Where $W_{t}$ is the Wiener process. The solution can be used to iteratively generate forecasts of a time series.

The $\mathrm{R}$ code for generating forecasts using this exact solution is as under:

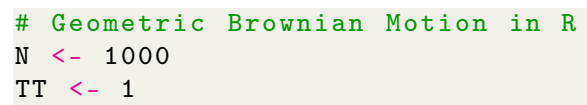




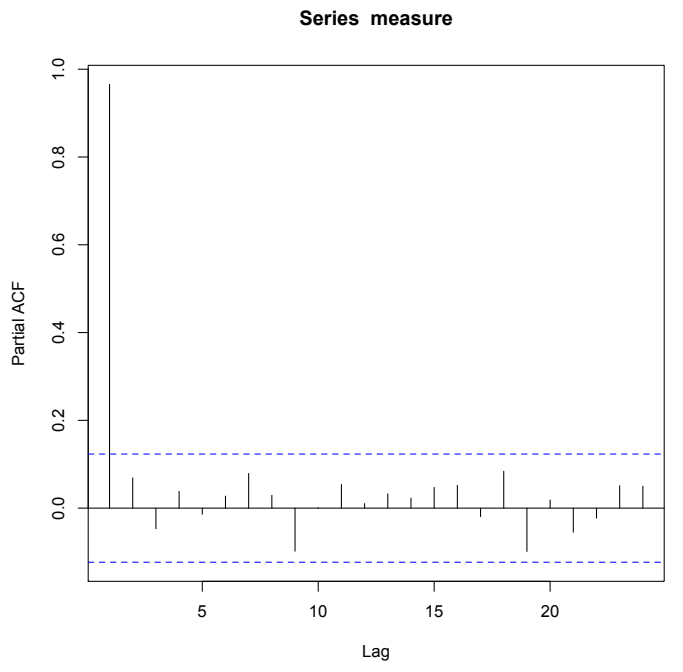

Figure 2: MSFT PACF Plot

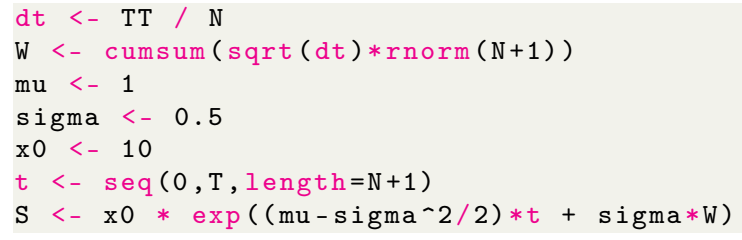

\subsection{Time-Dependent Non-linear Geometric Brownian Mo- tion (TDNGBM)}

In this algorithm, we have time dependent drift and time dependent diffusion parameters. We also have some non-linear terms.

The stochastic differential equation model is as follows:

$d X_{t}=\left(\theta_{1} X_{t}+\theta_{2} X_{t}^{2}+\theta_{3} t+\theta_{4} t^{2}\right) d t+\left(\theta_{5} X_{t}+\theta_{6} X_{t}^{2}+\theta_{7} t+\theta_{8} t^{2}\right) d B_{t}$

Where, $B_{t}$ is Brownian motion. The $\theta_{i}$ are learned using the Sim.DiffProc $\mathrm{R}$ package using maximum likelihood estimation.

\subsection{Bayesian Filter}

A Bayesian filter [6] is similar to a Kalman filter in that it does iterative updating and prediction using Bayesian updating. The current value is used as a 


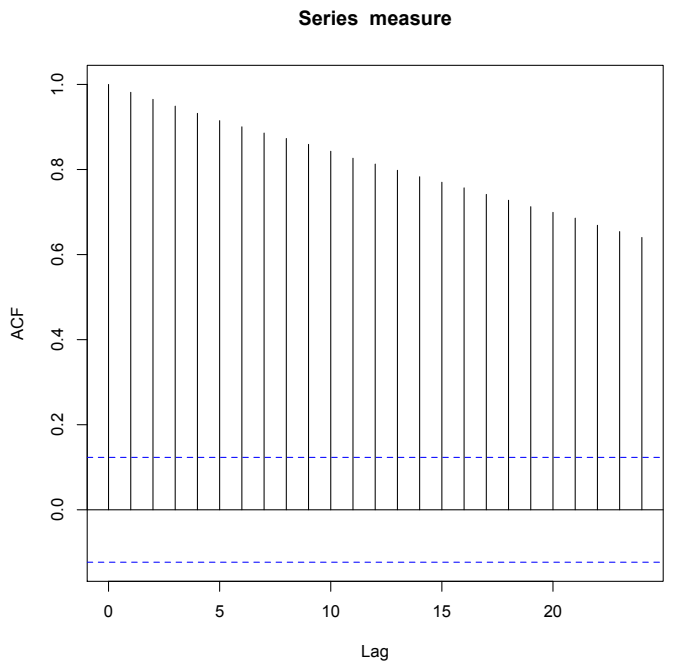

Figure 3: TSLA ACF Plot

prior to find the posterior mean of one future value. Then, that forecasted future value is used as a prior to create the second predicted mean (and variance) and so on. We predict 54 days in the future.

When the likelihood and the prior are Gaussian, the posterior is also Gaussian [9] [10].

If the likelihood is Gaussian $N\left(\mu, \sigma^{2}\right)$ and the prior is also Gaussian $N\left(\mu_{0}, \sigma_{0}^{2}\right)$, then the posterior is given by $N\left(\mu_{p}, \sigma_{p}^{2}\right)$

Where,

$\mu_{p}=\frac{\mu_{0} \sigma^{2}+\mu \sigma_{0}^{2}}{\sigma^{2}+\sigma_{0}^{2}}$

$\sigma_{p}^{2}=\frac{1}{\frac{1}{\sigma^{2}}+\frac{1}{\sigma_{0}^{2}}}$

These are the state update equations.

For prediction, the following is used:

$x_{n+1}=x_{n}+\Delta t \dot{x}_{n}$

$\Longrightarrow x_{n+1}=x_{n}+\Delta x_{n}$ (the motion equation [11] [10])

Two normal distributions are added, and thus their means and the variances 


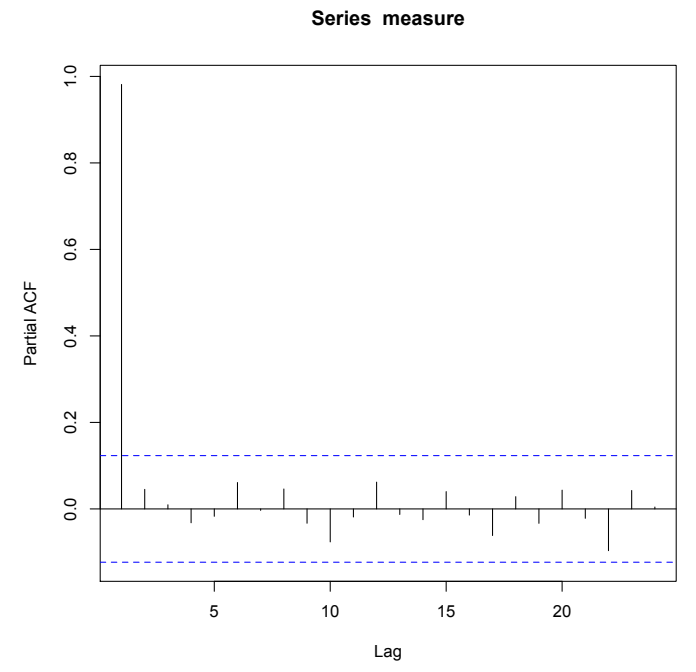

Figure 4: TSLA PACF Plot

are added to form the predictive distribution on $x_{n+1}[11]$.

\subsection{ARIMA}

Auto-regressive integrated moving average (ARIMA) [12] is a model to do forecasting on a time series. It has three components. One, an auto-regressive component that uses the history of stock prices to predict the future stock prices using AR techniques. Two, a moving average term that provides smoothing. And three, the integrated term that does differencing. We use the forecast package in $\mathrm{R}$.

\subsection{Kalman Filter}

The Kalman filter [8] is similar to the Bayesian filter [6] [10], in that it has prediction/correction updates. The Kalman filter has a value called the Kalman gain which has the weight of a measurement as compared to the weight of an estimate.

The Kalman gain $K_{n}=\frac{\text { EstimateVariance }}{\text { EstimateVariance+MeasurementVariance }}=\frac{p_{n-1}}{p_{n-1}+r}$

If the measurement variance is large, less weight is put on the measurements and more on the smoothed estimates. If the estimate uncertainty is large, more weight is placed on the measurements. As more data is collected, the estimates converge on a value close to the true smoothed values. 


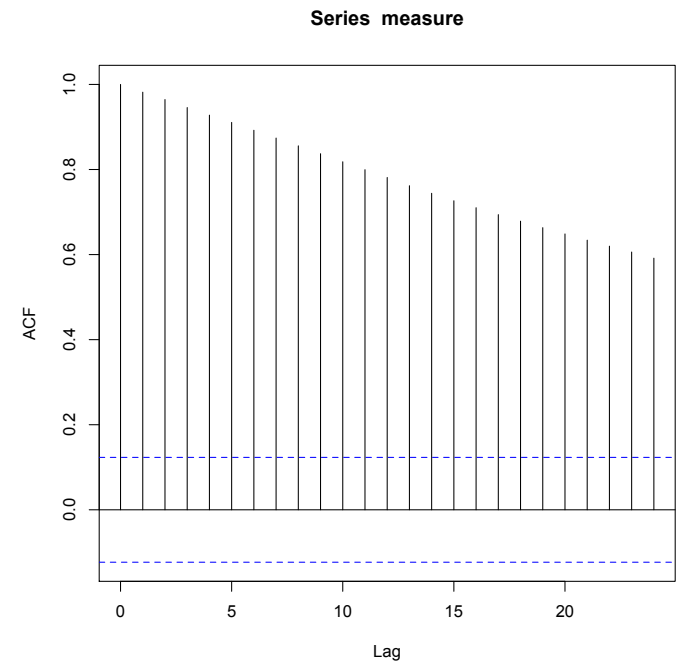

Figure 5: TGT ACF Plot

The state update equation is given as follows:

$x_{n}=\left(1-K_{n}\right) x_{n-1}+K_{n} z_{n}$

Where $x_{n}$ is the smoothed estimate and $z_{n}$ is the measurement.

(To understand the derivation of this equation, we suggest starting with chapter 2 in this book [13)

The measurement uncertainty does not change, but the estimate uncertainty $p_{n}$ changes using the following equation:

$p_{n}=\left(1-K_{n}\right) p_{n-1}$

Thus, as measurements come in, the Kalman filter updates the states and generates smoothed estimates. It calculates a new Kalman gain each iteration, and updates the estimate uncertainty, and then generates more smoothed estimates as more data comes in.

A good explanation of the Kalman filter in 1-dimensions is given here.

We use this library (KalmanLike) in the stats package in $\mathrm{R}$ for Kalman filter [8] forecasts. 


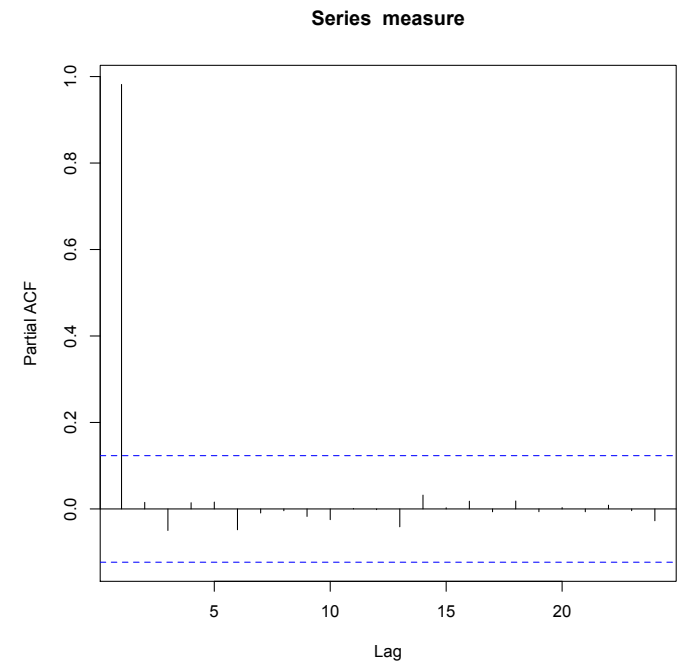

Figure 6: TGT PACF Plot

\subsection{Dynamic Harmonic Regression (DHR)}

Dynamic harmonic regression (DHR) [13 [14 is a dynamic forecasting technique suited to modeling time series with cyclical and seasonal periodicity. To learn the cyclical and seasonal components, it resorts to frequency domain optimization.

The DHR model looks like the following:

$y(t)=T(t)+C(t)+S(t)+\epsilon(t)$

Where, $T$ represents the trend, $C$ is the cyclical term and $S$ is the seasonal term.

$S(t)=\sum a_{i t} \cos \left(\omega_{i} t\right)+b_{i t} \sin \left(\omega_{i} t\right)$

$C(t)=\sum \alpha_{i t} \cos \left(f_{i} t\right)+\beta_{i t} \sin \left(f_{i} t\right)$

All parameters of the model are time dependent. As expected, this model is more suited for time series that have time dependent cycles and time dependent seasonality, like an increasing or decreasing trend with cycles.

The CAPTAIN toolbox of MATLAB [15] has the DHR implementation and contains several other implementations including the dynamic linear regression model which is based on ideas in this book [6]. We just mention this method 
Table 2: Results: Correlation Coefficients between the test set and the forecasts

\begin{tabular}{lllll}
\hline Method & TSLA & MSFT & TGT & Mean \\
\hline TDNGBM (100 sims) & 0.76 & 0.11 & -0.85 & $\mathbf{0 . 0 7}$ \\
\hline Bayesian Filter & 0.72 & 0.07 & -0.93 & -4.67 \\
\hline GBM & -0.78 & -0.72 & $\mathbf{0 . 2 1}$ & -0.43 \\
\hline ARIMA & -0.48 & -0.04 & -0.01 & -0.18 \\
\hline Kalman Filter & 0.72 & 0.07 & -0.93 & -0.05 \\
\hline
\end{tabular}

Table 3: Results: Mean Absolute Error (MAE) between the test set and the forecasts

\begin{tabular}{lllll}
\hline Method & TSLA & MSFT & TGT & Mean \\
\hline TDNGBM (100 sims) & 43.14 & $\mathbf{5 . 4 8}$ & 14.80 & $\mathbf{2 1 . 1 4}$ \\
\hline Bayesian Filter & $\mathbf{4 0 . 5 3}$ & 14.57 & 18.96 & 24.69 \\
\hline GBM & 56.13 & 5.85 & $\mathbf{1 3 . 4 7}$ & 25.15 \\
\hline ARIMA & 66.32 & 6.79 & 18.66 & 30.59 \\
\hline Kalman Filter & 40.90 & 15.98 & 19.79 & 25.56 \\
\hline
\end{tabular}

for completeness but we do not do any experiments with it.

\section{Results}

Results are shown in table 2. Table 2 shows the correlation between the actual test set values (54 days into the future) and the predicted values. Figures 7 through 12 show the dynamics of the processes as forecasted into the 54 days horizon using TDNGBM and ARIMA. Charts for the other algorithms can be generated using the code in our repository which also contains the data that is used here. Table 3 shows the MAE (mean absolute error) between the test set and the forecasted values.

As shown in table 2, the Time Dependent Non-linear Geometric Brownian Motion (TDNGBM) algorithm performs well on TSLA and MSFT, but does poorly on TGT. As shown in table 3, the MAE is better for TDNGBM, GBM and the Bayesian filter, each outperforming the rest of the algorithms in one of the stocks.

Confidence intervals on the estimates are more important than point estimates. As shown in figures 7,8 and 9, the confidence bounds of TDNGBM look pretty good and tight. Considering correlation or the MAE, TDNGBM does pretty well overall as compared to the other methods. 


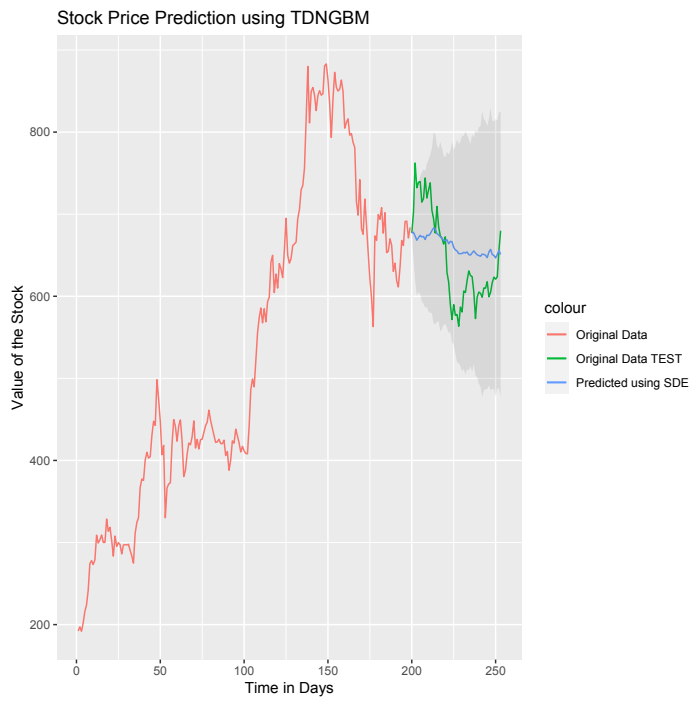

Figure 7: TSLA TDNGBM (100 simulations)

\section{Conclusion}

In this work, we compared five stochastic forecasting methods to predict the stock prices of Tesla, Microsoft and Target. There was no particular reason for choosing these three stocks. Results show that TDNGBM does reasonably well in terms of the MAE, correlation and the confidence intervals.

When considering the mean absolute error (MAE), TDNGBM, the Bayesian filter, and GBM outperform the others in one of the stocks.

Forecasting 54 days ahead is not easy for any method, and it might be useful to see how the algorithms do on a shorter horizon, like maybe 2 or 5 days.

While predicting stock prices, it appears that it might be useful to take several models and then average, or weighted average the values and that might turn out to be a highly accurate ensemble. Future work could include this, and also more hyper-parameter tuning of the models. For instance, it was noticed that transforming the time series data by using a logarithm improves the bounds for some of the stocks that we have not reported here.

\section{References}

[1] Bernt Oksendal. Stochastic differential equations: an introduction with applications. Springer Science \& Business Media, 2013. 


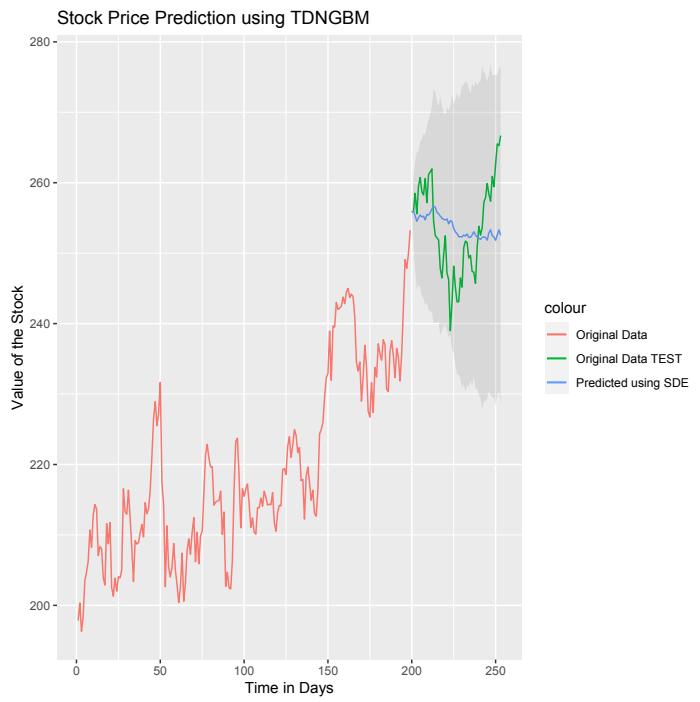

Figure 8: MSFT TDNGBM (100 simulations)

[2] Mohammad Rafiqul Islam and Nguyet Nguyen. Comparison of financial models for stock price prediction. Journal of Risk and Financial Management, 13(8):181, 2020.

[3] Harold Edwin Hurst. Long-term storage capacity of reservoirs. Transactions of the American society of civil engineers, 116(1):770-799, 1951.

[4] Sheldon M Ross. Introduction to probability models. Academic press, 2014.

[5] R Straja Sorin. Stochastic modeling of stock prices. Mongometry investment technology Inc, 200:1-19.

[6] Mike West and Jeff Harrison. Bayesian forecasting and dynamic models. Springer Science \& Business Media, 2006.

[7] Simo Särkkä. Bayesian filtering and smoothing. Number 3. Cambridge University Press, 2013.

[8] Rudolph Emil Kalman. A new approach to linear filtering and prediction problems. 1960.

[9] Wei Zhu. Bayesian inference for the normal distribution.

[10] Learning Machines. Kalman filter as a form of bayesian updating.

[11] Roger Labbe. Kalman and bayesian filters in python. Chap, 7(246):4, 2014.

[12] George EP Box, Gwilym M Jenkins, Gregory C Reinsel, and Greta M Ljung. Time series analysis: forecasting and control. John Wiley \& Sons, 2015 . 


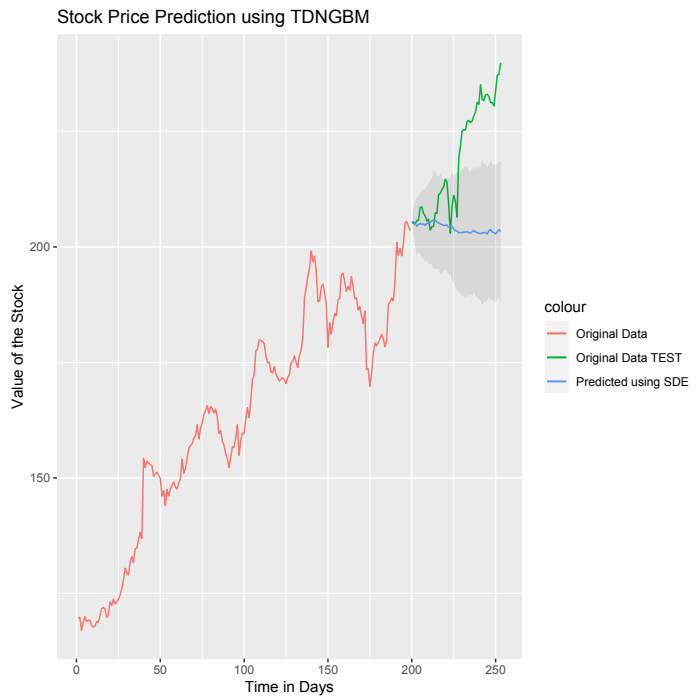

Figure 9: TGT TDNGBM (100 simulations)

[13] Peter C Young. Recursive estimation and time-series analysis: An introduction for the student and practitioner. Springer Science \& Business Media, 2011.

[14] Peter C Young, Diego J Pedregal, and Wlodek Tych. Dynamic harmonic regression. Journal of forecasting, 18(6):369-394, 1999.

[15] Peter C Young, C James Taylor, Wlodek Tych, DJ Pedregal, and Paul G McKenna. The captain toolbox. 2010.

[16] Peter C Young, Wlodzimierz Tych, and C James Taylor. The captain toolbox for matlab. IFAC Proceedings Volumes, 42(10):758-763, 2009. 


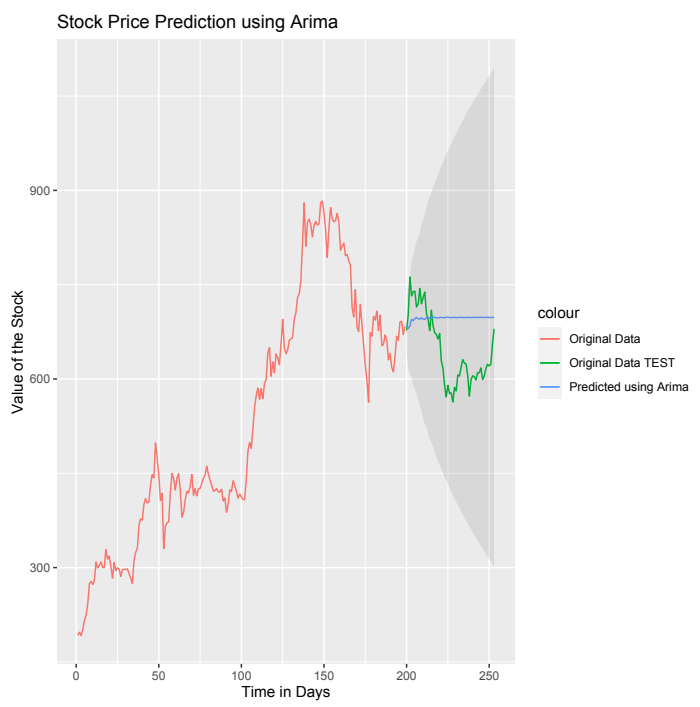

Figure 10: TSLA Arima

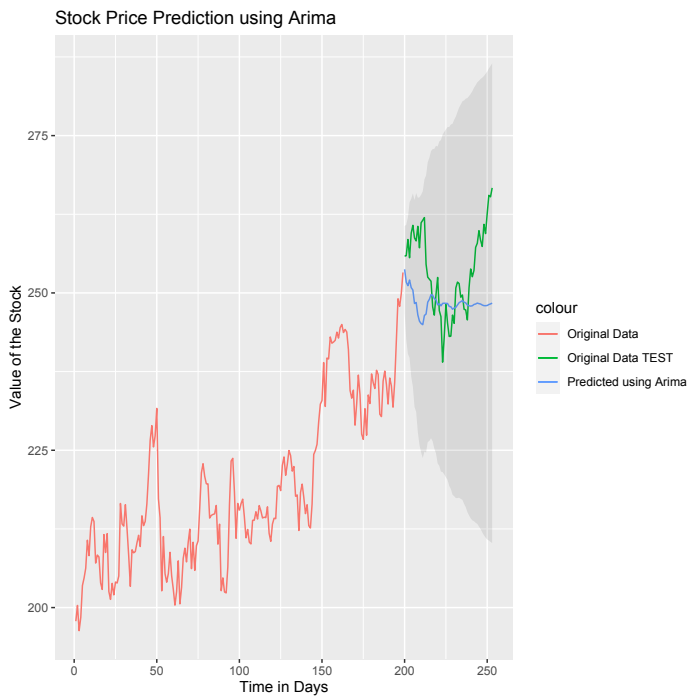

Figure 11: MSFT Arima 


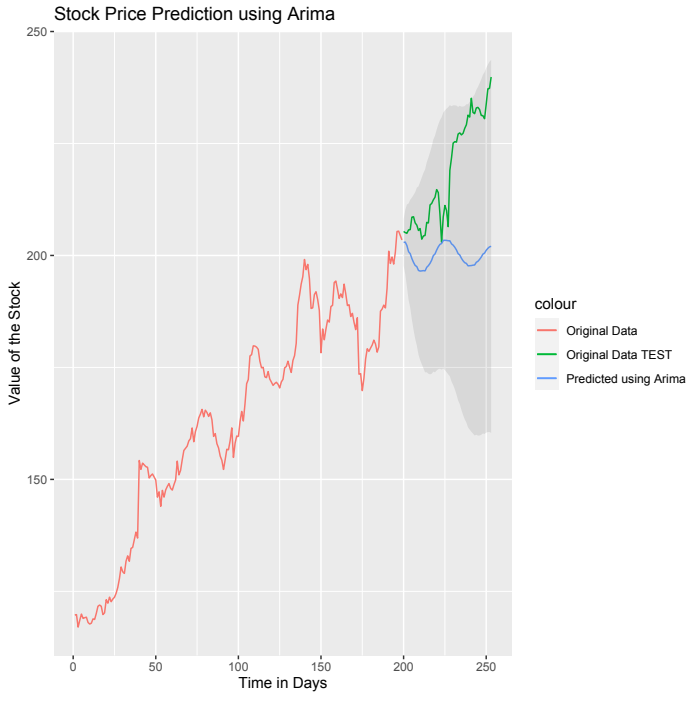

Figure 12: TGT Arima 